\title{
REVISIÓN DE LITERATURA SOBRE \\ EL APROVECHAMIENTO DE LA SEMILLA \\ DE MANGO PARA LA EXTRACCIÓN DE ACEITE \\ Y SUS POTENCIALES APLICACIONES \\ EN DIVERSAS INDUSTRIAS
}

\author{
Antonio Martin Córdova Córdova* \\ https://orcid.org/0000-0001-9258-6632 \\ Carlos Héctor Mendoza Mendoza* \\ https://orcid.org/0000-0001-6751-9454 \\ Universidad de Lima, Perú
}

Recibido: 30 de setiembre del 2020 / Aprobado: 29 de abril del 2021

doi: https://doi.org/10.26439/ing.ind2021.n41.5547

RESUMEN: La presente investigación tiene como objetivo demostrar, a través de una revisión de literatura, la existencia de aplicaciones industriales para el aceite de semilla de mango (ASM), puesto que cuenta con propiedades físico-químicas comparables e incluso mejores que otros aceites de semillas oleaginosas. Los artículos revisados indican que el ASM es comestible, de alta calidad, rico en materia insaponificable y constituido principalmente por los ácidos grasos esteárico y oleico. Asimismo, se revela el gran potencial del ASM como, por ejemplo, sustituto de la manteca de cacao, antioxidante natural, cosmético, compuesto antimicrobiano e, incluso, biocombustible. Estas aplicaciones permitirán a las industrias generar un mayor beneficio económico y contribuirán con el cuidado del medio ambiente.

PALABRAS CLAVE: aceite de semilla de mango / aprovechamiento semillas oleaginosas / revisión de literatura

\footnotetext{
*Correos electrónicos: 20140349@aloe.ulima.edu.pe; cmendoza@ulima.edu.pe
} 


\section{REVIEW OF LITERATURE ON THE USE OF MANGO SEED FOR OIL EXTRACTION AND ITS POTENTIAL APPLICATIONS IN VARIOUS INDUSTRIES}

ABSTRACT: This research aims to demonstrate through a review of literature the existence of industrial applications for mango seed oil (MSO) since it has comparable and even better physique-chemical properties than other oilseed oils. The revised articles indicate that the MSO is edible, of high quality, rich in unsaponifiable matter, and consisting mainly of stearic and oleic fatty acids. It also reveals the great potential of MSO as cocoa butter substitute, natural antioxidant, cosmetic, antimicrobial compound, and even biofuel. These applications will enable industries to generate more significant economic benefits and will contribute to the care of the environment.

KEYWORDS: mango seed oil / oilseed use / literature review 


\section{INTRODUCCIÓN}

El mango es una fruta tropical consumida en todo el mundo, ya sea directamente o como producto procesado (Torres-León et al., 2016). Su cáscara y hueso son descartados, lo que ocasiona contaminación ambiental debido a una incorrecta eliminación de desechos (Da Silva y Jorge, 2014). En el caso particular de las semillas, se estima que más de un millón de toneladas de estas son descartadas anualmente (Karunanithi et al., 2015).

El propósito de la presente investigación es responder la siguiente pregunta: ¿Existe el sustento necesario que demuestre que las propiedades del aceite de semilla de mango permiten su utilización en diferentes tipos de industria? Se consideró que la metodología de revisión de literatura permitirá responder a esta interrogante, pues esta se define como un escrito que agrupa un compendio de conocimientos relacionados a un tema específico y que tiene como propósito proveer un marco de referencia para justificar la importancia de un estudio (Creswell y Creswell, 2018).

\section{Información sobre el mango, la semilla y el aceite de semilla de mango}

El mango es una fruta oriunda de la India y del sudeste asiático (Fowomola, 2010), perteneciente al género Mangifera de la familia Anacardiaceae. Tal como indican Nzikou et al. (2010), la mayoría de los árboles frutales que se conocen comúnmente como mangos pertenecen a la especie Mangifera indica. Esta fruta cuenta con un sabor dulce, un aroma resinoso afrutado y puede encontrarse en tonalidades amarilla, naranja, verde y/o rojo (Sikdar et al., 2017). En la actualidad, el mango es una de las frutas más importantes entre las frutas tropicales, pues en el 2018 alcanzó el 52 \% de la producción mundial total de esta categoría de frutas que incluye a la piña, al aguacate y a la papaya (Organización de las Naciones Unidas para la Alimentación y la Agricultura, 2020).

El mango puede ser consumido como tal o procesado en productos congelados, enlatados, deshidratados o en bebidas listas para servir (Torres-León et al., 2016). Después del consumo o procesamiento industrial de la pulpa del mango, aproximadamente diez millones de toneladas de los demás componentes de esta fruta son descartados como residuo en todo el mundo (Sikdar et al., 2017). La utilización de subproductos de mango, especialmente de la semilla, puede ser una forma económica de reducir el problema de la eliminación de desechos generada por la industria procesadora de esta fruta (Kittiphoom, 2012). 
Tabla 1

Constitución del mango (según peso)

\begin{tabular}{ll}
\hline Componente & $\%$ \\
\hline Pulpa & $45-65$ \\
Cáscara & $12-15$ \\
Hueso o pepa & $10-25$ \\
\hline
\end{tabular}

Fuente: Karunanithi et al., (2015)

La constitución del mango y el porcentaje en peso de cada componente son indicados en la tabla 1. Se puede observar que el hueso constituye entre un 10 a $25 \%$ del peso total de la fruta en estudio, lo cual se corrobora con las investigaciones de TorresLeón et al. (2016) y Yadav et al. (2017). Ahora bien, en el interior de la pepa se encuentra la semilla del mango. Algunas de sus características físicas más representativas son su color amarillo y sus medidas de 4 a $7 \mathrm{~cm}$ de largo, 3 a $4 \mathrm{~cm}$ de ancho y $1 \mathrm{~cm}$ de espesor (Kittiphoom, 2012). En cuanto a sus características químicas, en la tabla 2 se puede observar que está compuesta principalmente por carbohidratos (58-80\%), grasa o aceite (6-16 \%) y proteínas (6-13\%). Respecto a la composición mineral y vitamínica, la semilla de mango contiene potasio, magnesio, fósforo, calcio, sodio y vitaminas $A, C$ y $E$ (Fowomola, 2010; Nzikou et al., 2010).

Tabla 2

Composición de la semilla de mango (en porcentajes)

\begin{tabular}{lcc}
\hline Componente & $\begin{array}{c}\text { Karunanithi et al. } \\
(2015)\end{array}$ & $\begin{array}{c}\text { Torres-León et al. } \\
(2016)\end{array}$ \\
\hline Proteína & 6,0 & $6-13$ \\
Grasa o aceite & 11,0 & $6-16$ \\
Carbohidratos & 77,0 & $58-80$ \\
\hline
\end{tabular}

\section{Elaboración propia}

Por otro lado, el aceite de semilla de mango (ASM), extraído a través de diversos métodos, y tal como se observa en la tabla 3, cuenta con un índice de yodo de 42,9-52,7 g/100 g aceite, un índice de saponificación de 189,9-190,7 mg KOH, y un punto de fusión de deslizamiento de $35,8-39,1^{\circ} \mathrm{C}$. Cabe mencionar que estas características son similares a las que presenta la grasa de semilla de cacao (Jahurul et al., 2015). 
Tabla 3

Propiedades químicas del aceite de semilla de mango

\begin{tabular}{lc}
\hline \multicolumn{1}{c}{ Propiedad química } & Torres-León et al. \\
& $(2016)$ \\
\hline Índice de yodo (g/100g aceite) & $42,9-52,7$ \\
Índice de saponificación (mg KOH) & $189,9-190,7$ \\
Punto de fusión de deslizamiento $\left({ }^{\circ} \mathrm{C}\right)$ & $35,8-39,1$ \\
\hline
\end{tabular}

Elaboración propia

Las investigaciones de Kittiphoom y Sutasinee (2013) y Nzikou et al. (2010) concuerdan en que la proporción de ácidos grasos insaturados es mayor que la de ácidos grasos saturados y que los principales ácidos grasos presentes en el ASM son el esteárico y el oleico (tabla 4). Debido a la presencia de estos dos ácidos grasos, se puede decir que el aceite de semilla de mango es químicamente más estable que otros aceites vegetales (Yadav et al., 2017).

Tabla 4

Ácidos grasos presentes en el aceite de semilla de mango (en porcentajes)

\begin{tabular}{lcc}
\hline \multicolumn{1}{c}{ Ácidos grasos } & $\begin{array}{c}\text { Kittiphoom y Sutasinee } \\
(2013)\end{array}$ & $\begin{array}{c}\text { Nzikou et al. } \\
(2010)\end{array}$ \\
\hline Saturados & 46,34 & 44,42 \\
esteárico & 37,37 & 37,94 \\
palmítico & 8,97 & 6,48 \\
Insaturados & 51,34 & 55,58 \\
oleico & 43,77 & 45,76 \\
linoleico & 6,78 & 7,45 \\
linolénico & 0,79 & 2,37 \\
\hline
\end{tabular}

Elaboración propia

La investigación de Karunanithi et al. (2015) precisa que el ASM es nutritivo, comestible y puede sustituir cualquier aceite sin efectos adversos. Ello lo corroboran Yadav et al. (2017) que sostienen que el índice de acidez del aceite en estudio $(4,77 \mathrm{mg} \mathrm{NaOH} / \mathrm{g})$ es un indicador de ser comestible e idóneo para su uso en la industria cosmética por su gran cantidad de materia insaponificable. Por otro lado, los bajos valores en ácidos grasos libres y peróxido presentes en el ASM son indicadores de su calidad y su posible uso directo en las industrias sin necesidad de pasar por un proceso de neutralización (Kittiphoom, 2012). 


\section{Métodos de extracción de aceite}

Los métodos de extracción que se utilizan en la actualidad para la extracción de aceite a partir de semillas oleaginosas son los que se muestran en la figura 1.

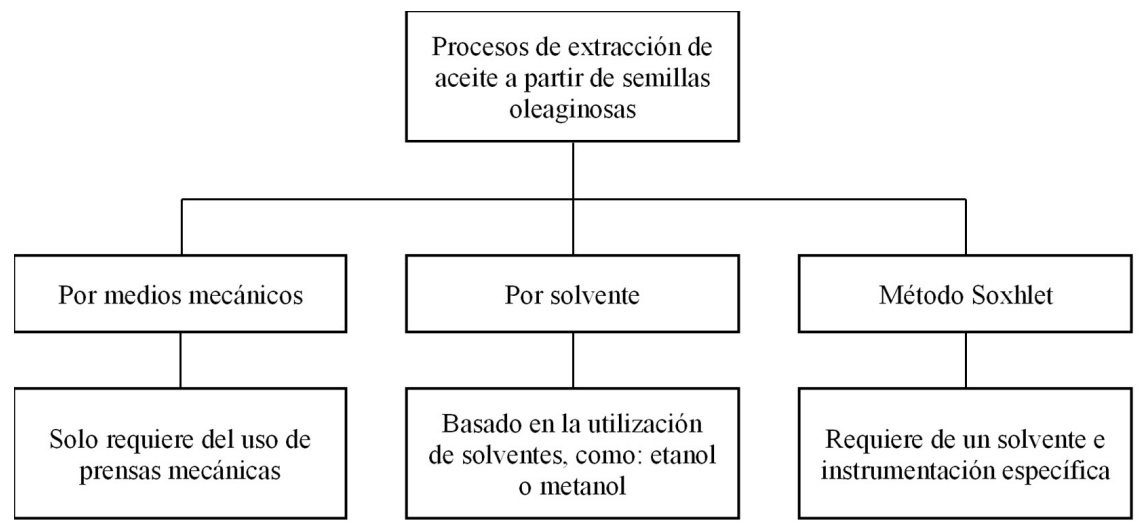

Figura 1. Procesos de extracción de aceite a partir de semillas oleaginosas

Elaboración propia

A continuación, se explica brevemente la operación de cada uno de estos métodos.

- Proceso por medios mecánicos

También conocido como el "método tradicional", es una técnica primitiva y simple que requiere el uso de prensas mecánicas que aplican presión sobre la masa de semillas a fin de obtener la mayor cantidad de aceite. El proceso empieza con el lavado de las semillas, luego continúa con la molienda, después con el prensado, de donde se obtienen dos productos: el aceite y la torta de semillas. Finalmente, se realiza un filtrado y tamizado de ambos productos (Tabio et al., 2017).

- Proceso por solvente

Método que consiste en la utilización de un solvente que permite la extracción del aceite de semillas oleaginosas. Es similar al proceso por medios mecánicos, con la salvedad de que las semillas son sometidas a duchas de solvente que arrastran el aceite a contracorriente, lo que permite extraerlo. Asimismo, se obtiene un subproducto llamado miscela, mezcla de aceite con solvente, que se somete a un proceso de filtrado y destilado para separar totalmente esta mezcla y recuperar la mayor cantidad de aceite (Sikdar et al., 2017). A continuación, en la tabla 5 se muestran los rendimientos obtenidos al utilizar este método con solventes distintos. Como se aprecia, el solvente n-hexano (15,2\%) presenta un rendimiento ligeramente más alto que el metanol (13,3\%). 
Tabla 5

Proceso por solvente: Solvente utilizado y rendimiento obtenido

\begin{tabular}{lll}
\hline & Nzikou et al., 2010 & Yadav et al., 2017 \\
\hline Solvente utilizado & metanol & n-hexano \\
Rendimiento obtenido (\%) & 13,3 & 15,2 \\
\hline
\end{tabular}

Elaboración propia

- Método Soxhlet

Es también un método por solvente pero que requiere de instrumentación específica. Está considerado como el método que genera el mayor rendimiento en la obtención de aceite por la recirculación del solvente que permite el aparato Soxhlet (Nzikou et al., 2010). Este conjunto instrumental comprende un matraz de fondo redondo, un extractor, un condensador, un calentador y un reciclador de agua (Sikdar et al., 2017).

Este proceso consiste en: primero, calentar el solvente líquido ubicado en el matraz; segundo, condensar la fase gaseosa del solvente; y tercero, precipitar las gotas de solvente condensado sobre el recipiente que contiene la masa de semillas de la cual se quiere extraer el aceite. Una vez lleno el recipiente, la mezcla solvente-aceite cae por gravedad nuevamente al matraz. Durante todo el proceso solo el solvente se irá evaporando, pero no el aceite que se irá obteniendo (Tabio et al., 2017).

\section{Antecedentes}

No se han encontrado revisiones de literatura recientes sobre este tema en particular; sin embargo, existen diversas investigaciones que se han desarrollado a través de los años que confirman el gran potencial que tiene la semilla de mango para su utilización en diversas industrias, tales como la cosmética, la farmacéutica, la alimentaria, entre otras (Jahurul et al., 2014; Kittiphoom, 2012). Por otro lado, Do Nascimento et al. (2019) indican que el proceso de extracción de aceite a partir de semillas de mango no ha sido explorado en la medida deseada debido al conocimiento limitado de las propiedades de la semilla de mango y las tecnologías de procesamiento adecuadas.

\section{METODOLOGÍA}

Para la realización de la presente investigación sobre el aprovechamiento de la semilla de mango para la extracción de aceite y sus potenciales aplicaciones en diversas industrias se utilizó como método la revisión de literatura. 
Las preguntas que guiaron el presente estudio fueron las siguientes: ¿existen investigaciones que identifiquen y sustenten las propiedades de la semilla del mango? y ¿existen investigaciones que identifiquen y sustenten las diferentes aplicaciones que puede tener el aceite de semilla de mango en la industria?

Criterios de búsqueda, inclusión / exclusión y selección de documentos

Se exploraron 20 artículos académicos en inglés y español extraídos de las bases de datos Scopus, Web of Science, Proquest y EBSCO. Para la búsqueda se utilizaron los conectores AND / OR y se tomaron en cuenta el título, resumen y palabras clave. El alcance geográfico de las referencias abarcó los continentes de Asia, África, América y Europa. Los criterios de selección utilizados fueron los siguientes:

- Artículos publicados en revistas indexadas y a texto completo

- Año de publicación con antigüedad no mayor a 10 años.

- Comprobación cuantitativa y/o cualitativa

- Referencias utilizadas

- No se incluyen investigaciones sobre las propiedades y utilización de la pulpa o de la cáscara del mango, solo se toman en cuenta las referidas a la semilla del mango.

En la tabla 6 se muestra un resumen de los artículos explorados y clasificados según la aplicación a la que corresponden. En el anexo, se encuentra la tabla completa donde se presenta cada investigación considerada y su respectiva clasificación.

Tabla 6

Relación de artículos agrupados por aplicación

\begin{tabular}{ll}
\hline Aplicación & Conteo \\
\hline A. Compuestos antimicrobianos para las industrias alimentaria y farmacéutica & 8 \\
B. Antioxidante natural para la industria alimentaria & 7 \\
C. Sustituto de la manteca de cacao & 4 \\
D. Cosmética & 4 \\
E. Producción de biodiesel & 3 \\
\hline
\end{tabular}

Elaboración propia 


\section{RESULTADOS - APLICACIONES DEL ASM EN LA INDUSTRIA}

\section{A. Compuestos antimicrobianos para las industrias alimentaria y farmacéutica}

En la actualidad, existe una fuerte tendencia respecto a la producción de compuestos antimicrobianos que pueden beneficiar a distintas industrias, entre las cuales las más relevantes son la alimentaria y la farmacéutica, debido a que estos sectores manejan productos perecibles y pueden ser atacados por bacterias. Debido a ello, se han publicado diversas investigaciones que señalan el potencial que tiene el ASM para esta aplicación.

- Awad El-Gied, A., Abdelkareem, A., y Hamedelniel, E. (2015). Investigation of Cream and Ointment on Antimicrobial Activity of Mangifera indica Extract.

Este artículo muestra que el ASM tiene un alto potencial como agente antimicrobiano cuando se formula como pomadas y cremas de uso tópico y podría, por tanto, explicar los éxitos que se alegan en el uso popular de la planta en el tratamiento de afecciones cutáneas comunes. Se prepararon diferentes lotes de formulación, entre los cuales la formulación de pomada (F03) mostró la mayor zona de inhibición contra el S. aureus, C. albicans, E. coli y M. smegmatis. Las formulaciones denominadas FCn (cremas) obtuvieron una zona de inhibición menor en comparación con las FOn (pomadas). Las formulaciones mostraron propiedades físicas aceptables y, por tanto, compatibles con la piel. Además, superaron la estabilidad a corto plazo, lo que indica la estabilidad física y química del producto. Por lo tanto, las formulaciones fueron portadores seguros y eficientes, con una potente actividad antimicrobiana.

- Sathi Reddy, K., Yahya Khan, M., Archana, K., Gopal Reddy, M., y Hameeda, B. (2016). Utilization of Mango Kernel Oil for the Rhamnolipid Production by Pseudomonas aeruginosa DR1 towards its Application as Biocontrol Agent.

En este caso se estudiaron el ASM y los ácidos grasos sintéticos (ácido palmítico, esteárico y oleico) para su uso como sustratos para la producción de ramnolípidos. Los resultados revelaron que el ASM es una fuente de carbono eficaz para la producción de ramnolípidos. Por lo cual, el ASM puede ser una potencial fuente de carbono renovable para la producción de biosurfactantes de ramnolípidos utilizando Paeruginosa DR1, y podría representar una alternativa ecológica a productos agroquímicos nocivos.

- Kim, H., Banerjee, N., Barnes, R. C., Pfent, C. M., Talcott, S. T., Dashwood, R. H., y Mertens-Talcott, S. U. (2016). Mango Polyphenolics Reduce Inflammation in Intestinal Colitis-Involvement of the miR-126/PI3K/AKT/mTOR Axis in vitro and in vivo. 
Este artículo trata de dilucidar los mecanismos subyacentes del efecto antiinflamatorio de los polifenoles del ASM en células CCD-18Co tratadas con lipopolisacáridos (LPS). Los resultados obtenidos sugieren que los polifenoles del ASM atenúan la respuesta inflamatoria tanto in vitro como in vivo; y por tanto, los polifenoles del mango podrían ser relevantes como agentes preventivos en la colitis ulcerosa.

- Rodríguez, E., Salangad, O., Almeda, R., Reyes, C., y Salamanez, K. (2019). Fatty Acid and Unsaponifiable Composition of Ten Philippine Food Plant Oils for Possible Nutraceutical and Cosmeceutical Applications.

Como parte del esfuerzo por maximizar la utilidad de plantas fácilmente disponibles e infrautilizadas en la agricultura, así como la de sus semillas, se investigó la composición de ácidos grasos e insaponificables de varios vegetales alimentarios. Los resultados mostraron que el aceite de almendra de aguacate contiene la fracción insaponificable más alta (72,63\%), seguido por el $\operatorname{ASM}(53,63$ - 59,29 \%). Al analizar la composición de la fracción insaponificable, el ASM presentó el mayor contenido en fitoesteroles. Los fitoesteroles poseen propiedades antitumorales, reductoras del colesterol, antioxidantes, antiinflamatorias y antibacterianas. Este estudio es novedoso por el análisis de las composiciones insaponificables y de ácidos grasos de diez aceites de plantas alimenticias de Filipinas y la comparación de los aceites mediante el análisis multidiscriminante. Los resultados indican que los diez materiales vegetales pueden ser fuentes de ingredientes funcionales e innovadores que pueden utilizarse para aplicaciones nutracéuticas y cosmecéuticas.

- Torres-León, C., Rojas, R., Contreras-Esquivel, J. C., Serna-Cock, L., BelmaresCerda, R. E., y Aguilar, C. N. (2016). Mango Seed: Functional and Nutritional Properties.

Esta investigación indica que el ASM cuenta con una excelente capacidad antimicrobiana y antidiarreica debido a su composición rica en antioxidantes y taninos. Asimismo, se señala que la materia prima a partir de la cual se obtiene el ASM, es un residuo biológico con un alto contenido de compuestos bioactivos (compuestos fenólicos, carotenoides, vitamina C y fibra dietética), los cuales mejoran la salud humana.

\section{B. Antioxidante natural para la industria alimentaria}

La calidad de las grasas y los aceites se deteriora principalmente por la autooxidación, que conduce a la pérdida de ácidos grasos esenciales y vitaminas, y genera efectos negativos en los alimentos (Turner et al., 2006). La autooxidación conduce a la generación 
de productos de oxidación tóxicos, que han sido implicados en el daño biológico de los tejidos y en el riesgo elevado de enfermedades cardiovasculares (Totani et al., 2008). Los antioxidantes son sustancias químicamente activas que pueden inhibir la autooxidación en grasas y aceites. La creciente preocupación por la toxicidad de los antioxidantes sintéticos ha llevado a explorar el potencial de los antioxidantes naturales para la estabilización de grasas y aceites.

- $\quad$ Nadeem, M., Imran, M., Iqbal, Z., Abbas, N., y Mahmud, A. (2016). Enhancement of the Oxidative Stability of Butter Oil by Blending with Mango (Mangifera indica L.) Kernel Oil in Ambient and Accelerated Oxidation.

La mezcla de manteca con ASM en todos los niveles mejoró significativamente la estabilidad oxidativa en ambientes de oxidación acelerada. El aceite de mantequilla complementado con ASM produjo una menor concentración de productos de oxidación primarios y secundarios con cambios menos pronunciados en la composición de ácidos grasos respecto al control. Estos resultados sugieren que la estabilidad de almacenamiento de manteca puede mejorarse mezclándola con ASM.

- Maryam Adilah, Z., Jamilah, B., y Nur Hanani, Z. (2018). Functional and Antioxidant Properties of Protein-Based Films Incorporated with Mango Kernel Extract for Active Packaging.

Esta investigación se centra en el desarrollo de envases activos mediante el uso de subproductos de la industria alimentaria. Se utilizó la proteína de soja (PS) y la gelatina de pescado (GP) como fuentes de biopolímeros y se añadieron diferentes concentraciones de ASM (del 1 al $5 \%$ ) como antioxidantes naturales, para evaluar las propiedades físicas, mecánicas y antioxidantes de las películas de PS y GP.

La incorporación de ASM en las películas de PS y GP aumentó significativamente el espesor, la resistencia a la tracción y la actividad antioxidante. En cambio, el ASM redujo la solubilidad en agua y el alargamiento a la rotura. Las películas GP mostraron un mayor grosor, resistencia a la tracción y transparencia. Sin embargo, la película de PS mostró una mejor actividad antioxidante que la película de GP. Tanto las películas de GP como las de PS incorporadas con ASM mostraron un gran potencial para ser desarrolladas como películas antioxidantes.

- Torres-León, C., Ramírez-Guzmán, N., Ascacio-Valdés, J., Serna-Cock, L., dos Santos Correia, M. T., Contreras-Esquivel, J. C., y Aguilar, C. N. (2019). SolidState Fermentation with Aspergillus niger to Enhance the Phenolic Contents and Antioxidative Activity of Mexican Mango Seed: A Promising Source of Natural Antioxidants. 
Este estudio examina los cambios en los compuestos fenólicos y la actividad antioxidante del ASM con el hongo Aspergillus niger GH1. La fermentación en estado sólido (FES) tiene un gran potencial para la obtención de compuestos bioactivos, ya que los microorganismos como los hongos producen naturalmente enzimas que degradan la pared celular, generando una hidrólisis y movilización de compuestos hacia el solvente de extracción. Este proceso biotecnológico es económico y fácil de aplicar, ya que requiere un equipo pequeño, un capital menor y unos costos operativos reducidos (Letti et al., 2017). La FES con el hongo Aspergillus niger $\mathrm{GH} 1$ resulta un método eficaz que aumenta significativamente el contenido de polifenoles en la semilla de mango Ataúlfo. El proceso de fermentación liberó los compuestos fenólicos que estaban unidos a la matriz vegetal, lo que se refleja en un aumento del contenido de fenoles libres. La fracción fenólica libre de la semilla de mango fermentada presentó una mayor actividad antioxidante que la semilla de mango sin fermentar. Ello se debe al alto contenido de compuestos polifenólicos, principalmente galotaninos. Los extractos (aceite) de semillas de mango fermentadas son especialmente prometedores como ingredientes funcionales para la industria alimentaria.

- Mwaurah, P. W., Kumar, S., Kumar, N., Panghal, A., Attkan, A. K., Singh, V. K., y Garg, M. K. (2020). Physicochemical Characteristics, Bioactive Compounds and Industrial Applications of Mango Kernel and its Products: A Review.

El análisis de la composición nutricional de la semilla de mango determina que es rica en proteínas (aminoácidos esenciales como la leucina, la valina, la isoleucina y la lisina, en diferentes proporciones en base al peso seco), minerales (calcio, magnesio y potasio), vitaminas (provitamina $A$, vitaminas $E, K, C$ y otras como B1, B2, B6 y B12 en menores proporciones), carbohidratos, lípidos y fitoquímicos. Por otro lado, el ASM tiene componentes bioactivos importantes, como compuestos antioxidantes, compuestos fenólicos (ácidos fenólicos y flavonoides de uso en la industria) y taninos; lo cual hace viable su mezcla con otros productos de origen vegetal o animal para la fabricación de alimentos.

- Torres-León, C., Rojas, R., Serna-Cock, L., Belmares-Cerda, R., y Aguilar, C. N. (2017). Extraction of Antioxidants from Mango Seed Kernel: Optimization Assisted by Microwave.

La semilla del mango es una fuente potencial de compuestos funcionales. Tiene un alto contenido de compuestos bioactivos (ácidos fenólicos, flavonoides, galotaninos y elagitaninos) que mejoran la salud humana (Dorta et al., 2014; Ribeiro y Schieber, 2010). Esta característica es particularmente relevante ante el aumento de demanda de productos naturales y el incremento de los efectos nocivos para la salud de los antioxidantes sintéticos (Lorenzo et al., 2013). En 
2008, el extracto de etanol de la semilla de mango fue catalogado como uno de los cuatro extractos alimentarios con mayor capacidad antioxidante, en comparación con el ácido L-ascórbico (Saito et al., 2008).

Para recuperar los compuestos bioactivos de los subproductos agroindustriales, deben considerarse los factores de control que afectan significativamente a la separación. Los métodos de extracción en los que se utilizan altas temperaturas reportan efectos negativos en la actividad antioxidante de los extractos de las semillas de mango. Para obtener extractos con alta actividad biológica, se propone la extracción asistida por microondas (EAM). La EAM presenta amplias ventajas en comparación con las técnicas de extracción convencionales, ya que ofrece mayores recuperaciones, menor tiempo de operación y permite el uso de una menor cantidad de disolvente, además de preservar la actividad antioxidante. Actualmente, la EAM es útil a escala industrial y ha despertado especial interés porque aumenta la viabilidad de los procesos y garantiza una mayor calidad del bioproducto (Cirimina et al., 2016).

Entre los factores de extracción, el método PBD (Plackett-Burman Design) determina la relación sólido-líquido, la temperatura y el ciclo de extracción como los factores significativos que influyen en la extracción de compuestos con alta actividad antioxidante. En estas condiciones, los extractos mostraron una elevada actividad antioxidante, contenido fenólico y actividad de barrido de radicales.

- Azeem, M., Nadeem, M., y Sajid, R. (2015). Analytical Characterization of Pure and Blended Watermelon (Citrulluslanatus) Oil: Impact of Blending on Oxidative Stability.

Este artículo presenta, de modo experimental, la utilización del ASM como agente a través del cual puede reducirse la presencia de ácidos grasos libres de las mezclas de aceite de sandía y ASM. Los ácidos grasos libres del aceite de sandía, que eran del $1,38 \%$, se redujeron al 0,82 \% cuando se añadió un $40 \%$ de ASM en el aceite de sandía. El índice de yodo de todas las mezclas disminuyó a raíz de la adición de ASM. El color y el valor de saponificación de las mezclas no fueron diferentes de los aceites de base, sin embargo, los índices de refracción e insaponificación fueron estadísticamente diferentes de los aceites de base. La mayor cantidad de materia insaponificable en el ASM estaba relacionada con los hidrocarburos, los esteroles, los fenoles, los carotenoides y los tocoferoles.

El ASM es superior a otros aceites en cuanto a una mayor estabilidad oxidativa, lo que puede explicarse por el mayor número de ácidos grasos monoinsaturados y compuestos fenólicos, que pueden actuar como inhibidores del mecanismo de los radicales libres. La caracterización del ASM reveló que los ácidos grasos principales eran el oleico y el esteárico. 


\section{Sustituto de la manteca de cacao}

La manteca de cacao (MC) se obtiene de la semilla de la fruta del mismo nombre. Salminen et al. (2020) precisan en su investigación que los ácidos grasos que constituyen principalmente a la $\mathrm{MC}$ son el ácido palmítico $\left(\mathrm{C}_{16: 0}, 25-27 \%\right)$, el ácido esteárico $\left(\mathrm{C}_{18: 0}, 33-38 \%\right)$, el ácido oleico $\left(\mathrm{C}_{18: 1}, 33-36 \%\right)$ y el ácido linoleico $\left(\mathrm{C}_{18: 2}, 2-4 \%\right)$. Además, cabe mencionar que el precio de la MC es de los más altos entre las grasas comerciales debido a su baja disponibilidad y alta demanda por parte de las industrias alimentaria, farmacéutica y cosmética (Kittiphoom, 2012). Por ende, en la actualidad, existe una búsqueda activa de sustitutos de esta grasa que se ve reflejada en diversos artículos científicos que platean diversos reemplazantes para la MC, entre los cuales se encuentra el ASM. A continuación, se exponen los principales aportes de tres artículos representativos seleccionados sobre la base de los criterios expuestos en el inciso correspondiente a la metodología de este artículo.

- Jahurul, M. H. A., Zaidul, I. S. M., Nik Norulaini, N. A., Sahena, F., Kamaruzzaman, B. Y., Ghafoor, K., y Omar, A. K. M. (2014). Cocoa Butter Replacers from Blends of Mango Seed Fat Extracted by Supercritical Carbon Dioxide and Palm Stearin.

En este artículo se indica que es posible crear sustitutos de la MC a partir de la mezcla de ASM con estearina de palma (EP) en distintas proporciones. Los resultados se sustentan en los valores obtenidos de las propiedades fisicoquímicas que se presentan en la tabla 7. Como se observa, los valores del índice de yodo de las distintas mezclas están por encima del valor de la MC. Ello indica que la MC cuenta con una mayor dureza. Por otro lado, el punto de fusión de deslizamiento de las mezclas está relacionado directamente con el contenido de EP y los valores obtenidos son mayores que los de la MC, lo cual quiere decir que las mezclas soportan una mayor temperatura antes de comenzar a derretirse. Finalmente, en cuanto al índice de saponificación, la diferencia entre las mezclas y la MC resulta ser mínima ( 1 \%). En conclusión, la mezcla de ASM y EP en la correcta proporción representa un sustituto factible de la MC.

Tabla 7

Propiedades de mezclas Aceite de semilla de mango (ASM) / Estearina de Palma (EP)

\begin{tabular}{|c|c|c|c|c|c|}
\hline \multirow[t]{2}{*}{ Propiedad } & \multicolumn{4}{|c|}{ Mezcla (ASM/EP) } & \multirow{2}{*}{$\begin{array}{c}\text { Manteca de cacao } \\
\text { (MC) }\end{array}$} \\
\hline & $90 / 10$ & $85 / 15$ & $80 / 20$ & $75 / 25$ & \\
\hline $\begin{array}{l}\text { Índice de yodo } \\
\text { (g } 12 \text { / 100g } \\
\text { grasa) }\end{array}$ & $42,4 \pm 0,7$ & $42,2 \pm 0,4$ & $41,9 \pm 0,8$ & $41,8 \pm 1,0$ & $34,0 \pm 0,47 a$ \\
\hline $\begin{array}{l}\text { Punto de fusión } \\
\text { de deslizamiento } \\
\left({ }^{\circ} \mathrm{C}\right)\end{array}$ & $37,7 \pm 0,2$ & $38,4 \pm 0,4$ & $39,2 \pm 0,3$ & $39,9 \pm 0,1$ & $27,8 \pm 0,0 \mathrm{a}$ \\
\hline
\end{tabular}


(continuación)

\begin{tabular}{llllll}
\hline Índice de & $195,7 \pm 1,0$ & $195,7 \pm 0,3$ & $195,8 \pm 0,5$ & $195,9 \pm 0,1$ & $190,7 \pm 1,13$ a \\
saponificación & & & \\
(mg KOH / g & & & \\
grasa) & & & \\
\hline
\end{tabular}

Fuente: Jahurul et al. (2014). a Sonwai et al. (2014)

- Jin, J., Warda, P., Qi, C., Sun, C., Jie, L., Xie, D., Huang, J., Jin, Q., y Wang, X. (2016). Mango Kernel Fat-Based Chocolate Fat with Heat Resistant Triacylglycerols: Production Via Blending Using Mango Kernel Fat Mid-Fraction and Palm Mid-Fractions Produced in Different Fractionation Paths.

A diferencia de la investigación anterior de Jahurul et al. (2014), esta se basa en la formulación de mezclas enriquecidas con triacilgliceroles resistentes al calor (TRC) de ASM fraccionado (ASMf) y aceite de palma fraccionado (APf) (ver figura 2) que puedan sustituir a la MC y que puedan tener una mayor resistencia al calor.

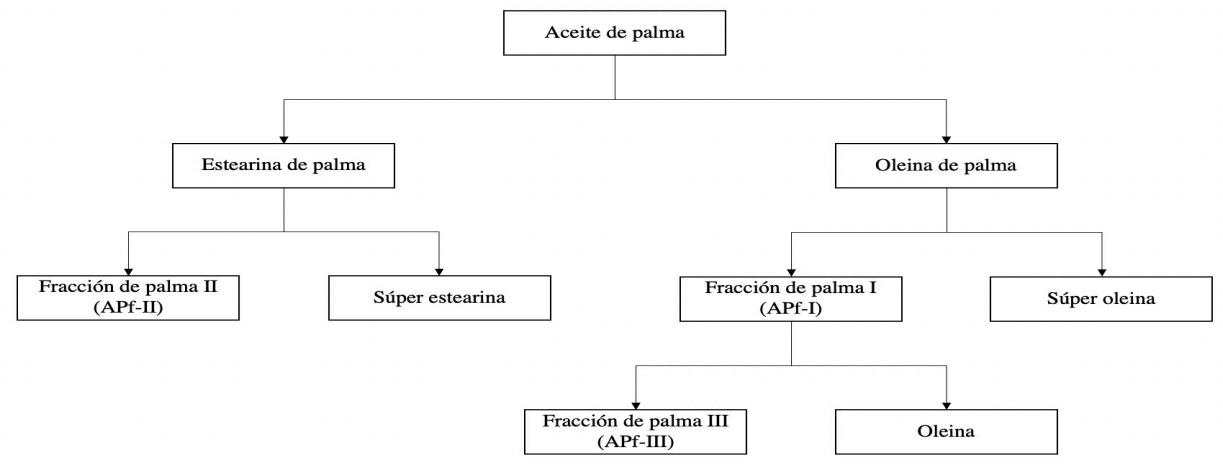

Figura 2. Productos del fraccionamiento por múltiples etapas del aceite de palma

Fuente: Jin et al. (2016)

En la tabla 8 se observa que el índice de yodo se incrementa con una mayor proporción de APf-I o APf-II en las respectivas mezclas, mientras que con aquellas mezclas que contienen APf-III sucede lo contrario. En cuanto al punto de fusión de deslizamiento, las mezclas ASMf/APf de proporción 90/10 respectivamente, indican una mejor resistencia al calor en comparación con la MC. Finalmente, los valores del contenido de grasa sólida a $20^{\circ} \mathrm{C}$ obtenidos indican que las mezclas ASMf/APf de proporción 90/10 respectivamente, también resultan ser las mejores en comparación con las demás mezclas y a la MC. En conclusión, es factible decir que las mezclas con ratio 90:10, de ASMf/APf 
respectivamente, demuestran tener mejores propiedades en comparación con la MC y, por ende, pueden sustituirla.

Tabla 8

Propiedades de mezclas ASMf/APf

\begin{tabular}{|c|c|c|c|}
\hline $\begin{array}{c}\text { Mezcla } \\
\text { (ASMf/APf) }\end{array}$ & $\begin{array}{l}\text { Índice de yodo } \\
\text { (g I2 / 100g grasa) }\end{array}$ & $\begin{array}{l}\text { Punto de fusión de desli- } \\
\text { zamiento }\left({ }^{\circ} \mathrm{C}\right)\end{array}$ & $\begin{array}{c}\text { Contenido de grasa sólida } \\
\text { a } 20{ }^{\circ} \mathrm{C}(\%)\end{array}$ \\
\hline $\begin{array}{l}\text { ASMf/APf-I } \\
90 / 10\end{array}$ & $35,0 \pm 1,3$ & $26,1 \pm 0,3$ & $\sim 84,0$ \\
\hline $\begin{array}{l}\text { ASMf/APf-I } \\
80 / 20\end{array}$ & $35,8 \pm 2,1$ & $25,9 \pm 0,5$ & $\sim 75,0$ \\
\hline $\begin{array}{c}\text { ASMf/APf-I } \\
70 / 30\end{array}$ & $36,2 \pm 2,1$ & $25,1 \pm 0,9$ & $\sim 67,0$ \\
\hline $\begin{array}{l}\text { ASMf/APf-II } \\
90 / 10\end{array}$ & $34,3 \pm 1,3$ & $29,3 \pm 0,5$ & $\sim 84,5$ \\
\hline $\begin{array}{l}\text { ASMf/APf-II } \\
80 / 20\end{array}$ & $34,9 \pm 1,0$ & $28,7 \pm 0,9$ & $\sim 77,5$ \\
\hline $\begin{array}{l}\text { ASMf/APf-II } \\
70 / 30\end{array}$ & $35,5 \pm 0,2$ & $28,1 \pm 1,1$ & $\sim 68,0$ \\
\hline $\begin{array}{l}\text { ASMf/APf-III } \\
90 / 10\end{array}$ & $33,2 \pm 0,6$ & $29,9 \pm 0,3$ & $\sim 86,0$ \\
\hline $\begin{array}{l}\text { ASMf/APf-III } \\
80 / 20\end{array}$ & $32,6 \pm 1,4$ & $28,9 \pm 1,0$ & $\sim 82,5$ \\
\hline $\begin{array}{l}\text { ASMf/APf-III } \\
70 / 30\end{array}$ & $32,0 \pm 0,1$ & $27,7 \pm 0,8$ & $\sim 79,0$ \\
\hline $\begin{array}{c}\text { Manteca de cacao } \\
\text { (MC) }\end{array}$ & $34,0 \pm 0,47 a$ & $25,3-26,1$ & $\sim 70,0$ \\
\hline
\end{tabular}

Fuente: Jin et al. (2016). a Sonwai et al. (2014)

- Jin, J., Mu, H., Wang, Y., Pembe, W., Liu, Y., Huang, J., Jin, Q., y Wang, X. (2016). Production of High-Melting Symmetrical Monounsaturated Triacylglycerol-Rich Fats from Mango Kernel Fat by Acetone Fractionation.

Esta investigación se basa en la formulación de mezclas de ASMf-II con MC, a fin de determinar si la GSMf-Il puede optimizar las propiedades térmicas de la MC. Primero, se realiza un estudio de las fracciones del ASM obtenidas del proceso de fraccionamiento de 3 etapas (ver figura 3). Luego de analizar diversas propiedades, tales como la composición de triacilgliceroles, índice de yodo, punto de fusión de deslizamiento, propiedades de fusión y cristalización, entre otras, se concluye que la fracción II resulta ser la mejor en comparación con las demás. 


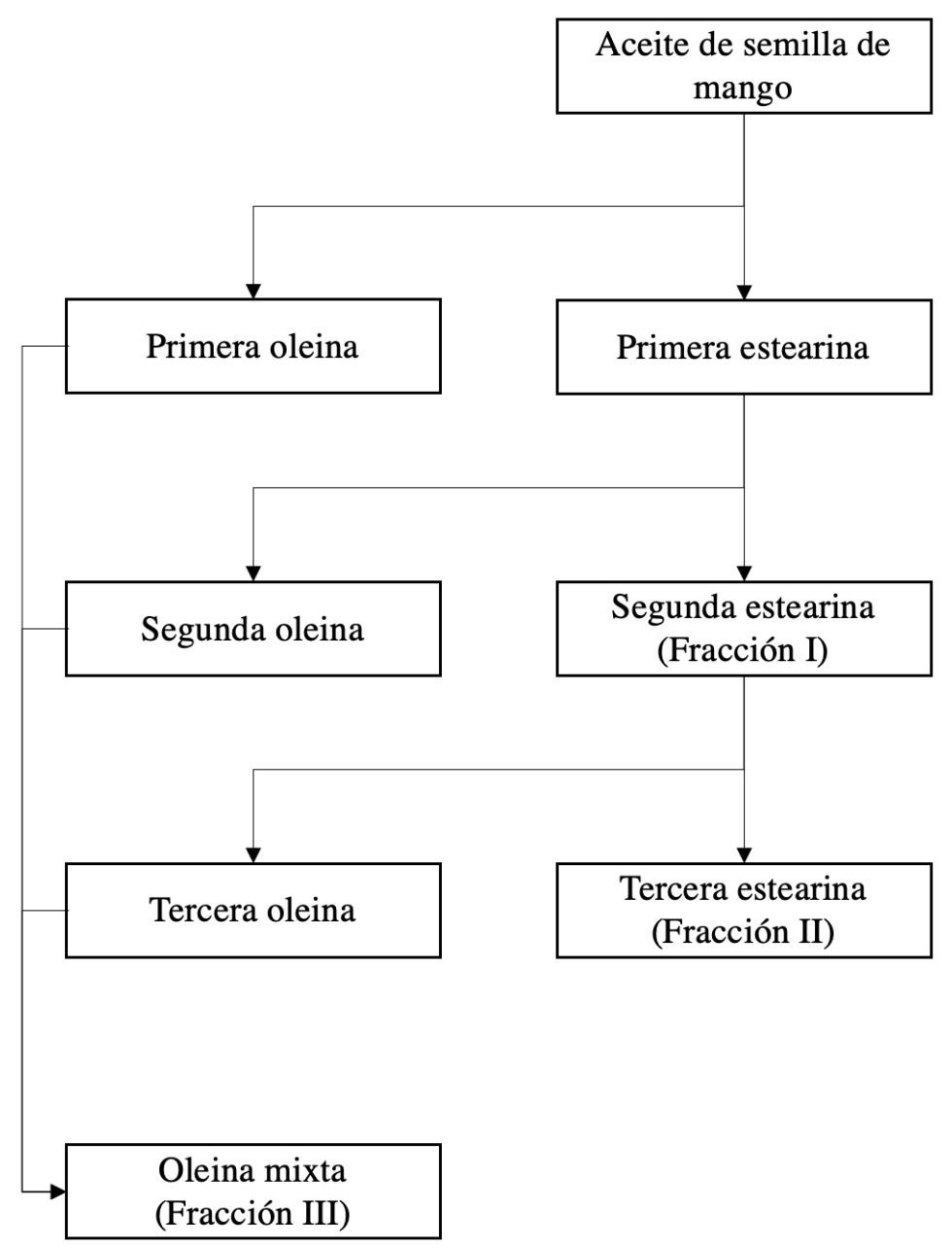

Figura 3. Productos del fraccionamiento por múltiples etapas del ASM

Fuente: Jin et al. (2016)

Después, se procede con la formulación de las mezclas de ASMf-II con MC en proporciones $15 / 85,30 / 70$ y $45 / 55$, respectivamente. Como se observa en las tablas 9 y 10 , las propiedades térmicas de las mezclas que constan de 30-45 \% de ASMf-II y $55-70 \%$ de MC presentan mejores propiedades que la MC. Por lo tanto, se concluye que la fracción II del ASM es adecuada para la elaboración de productos de confitería resistentes al calor. 
Tabla 9

Propiedad de fusión de las mezclas ASMf-II/MC

\begin{tabular}{ccccc}
\hline & $\begin{array}{c}\text { Temperatura de } \\
\text { inicio }\left({ }^{\circ} \mathrm{C}\right)\end{array}$ & $\begin{array}{r}\text { Temperatura } \\
\text { máxima }\left({ }^{\circ} \mathrm{C}\right)\end{array}$ & $\begin{array}{r}\text { Temperatura de compen- } \\
\text { sación }\left({ }^{\circ} \mathrm{C}\right)\end{array}$ & $\begin{array}{c}\text { Entalpía } \\
(\mathrm{J} / \mathrm{g})\end{array}$ \\
$\begin{array}{c}\text { ASMf-II/MC } \\
15 / 85\end{array}$ & $17,0 \pm 0,1$ & $21,9 \pm 0,6$ & $29,3 \pm 1,1$ & $75,1 \pm 1,2$ \\
ASMf-II/MC \\
$\begin{array}{l}\text { 30/70 } \\
\text { ASMf-II/MC } \\
\text { 45/55 }\end{array}$
\end{tabular}

Fuente: Jin et al. (2016)

Tabla 10

Propiedad de cristalización de las mezclas ASMf-II/MC

\begin{tabular}{ccccc}
\hline & $\begin{array}{c}\text { Temperatura } \\
\text { de inicio }\left({ }^{\circ} \mathrm{C}\right)\end{array}$ & $\begin{array}{c}\text { Temperatura } \\
\text { máxima }\left({ }^{\circ} \mathrm{C}\right)\end{array}$ & $\begin{array}{c}\text { Temperatura } \\
\text { de compensación }\left({ }^{\circ} \mathrm{C}\right)\end{array}$ & $\begin{array}{c}\text { Entalpía } \\
(\mathrm{J} / \mathrm{g})\end{array}$ \\
\hline $\begin{array}{c}\text { ASMf-II/MC } \\
15 / 85\end{array}$ & $18,9 \pm 0,3$ & $15,5 \pm 0,7$ & $-15,1 \pm 0,6$ & $71,3 \pm 1,2$ \\
$\begin{array}{c}\text { ASMf-II/MC } \\
\text { 30/70 }\end{array}$ & $19,4 \pm 0,6$ & $16,2 \pm 0,2$ & $-14,1 \pm 0,3$ & $74,9 \pm 1,6$ \\
$\begin{array}{c}\text { ASMf-II/MC } \\
\text { 45/55 }\end{array}$ & $20,2 \pm 0,7$ & $16,9 \pm 0,5$ & $-14,3 \pm 0,5$ & $75,8 \pm 0,3$ \\
$\begin{array}{l}\text { Manteca de } \\
\text { cacao (MC) }\end{array}$ & $17,3 \pm 1,0$ & $14,4 \pm 0,4$ & $-15,8 \pm 0,8$ & $69,1 \pm 1,5$ \\
\hline
\end{tabular}

Fuente: Jin et al. (2016).

\section{Cosmética}

Kittiphoom (2012) y Rodríguez et al. (2019) indican que el ASM cuenta con un gran potencial para ser utilizado como materia prima para la producción de diversos cosméticos, gracias a características como la cantidad de compuestos fenólicos, la cantidad de esteroles, el perfil de ácidos grasos, entre otras, que lo vuelven óptimo para ser un insumo alternativo viable para dicha industria. A continuación, se hace una revisión de dos artículos representativos que señalan la aplicación del aceite en estudio. 
- Wu, S., Tokuda, M., Kashiwagi, A., Henmi, A., Okada, Y., Tachibana, S., y Nomura, M. (2015). Evaluation of the Fatty Acid Composition of the Seeds of Mangifera indica L. and their Application.

Este estudio tiene como fin analizar la composición del ASM y producir experimentalmente cosméticos a partir de esta materia prima. Se encontró que los principales ácidos grasos presentes en el ASM son los ácidos oleico y esteárico; mientras que los triacilgliceroles fueron los lípidos hallados en mayor cantidad $(93,9-96,6 \%)$. Por otro lado, se produjeron lociones y jabones utilizando el ASM. En cuanto a las lociones, se señala que estas tienen una apariencia líquida viscosa y excelentes capacidades humectantes. Respecto a los jabones, se indica que estos cuentan con un $\mathrm{pH}$ similar a los jabones comerciales $(\sim 8,7)$ y alta capacidad detergente. Se concluye que el ASM tiene las propiedades adecuadas para poder producir diversos productos de la industria cosmética.

- Nahar, M. K., Lisa, S. A., Nada, K., y Begum, M. (2017). Characterization of Seed Kernel Oil of Bangladeshi Mango and its Evaluation as Cosmetic Ingredient.

Esta investigación tiene como propósito estudiar el contenido de aflatoxinas en el aceite de semilla de cuatro variedades de mango oriundas de Bangladesh. Las aflatoxinas son potentes carcinógenos y su contenido debe estar limitado según ciertos estándares establecidos. Los resultados indican que la cantidad promedio de aflatoxina B1 presente en estos aceites es de 1,46 ppb y la cantidad total de aflatoxinas es de $5,15 \mathrm{ppb}$, los cuales están dentro de los parámetros establecidos por el estándar de la Comunidad Europea ( $2 \mathrm{ppb}$ ) y la agencia estadounidense de Administración de Alimentos y Drogas (20 ppb), respectivamente. Por otro lado, estos aceites cumplen también con el parámetro de contenido de metales pesados, pues la cantidad de plomo $(0,45 \mathrm{ppm})$ y arsénico $(0,03 \mathrm{ppm})$ está dentro de lo que establece el Estándar Indio de la Grasa de Semilla de Mango como límites máximos ( $\mathrm{Pb} 5,0$ y As 0,5 ppm). Por ende, se concluye que estos aceites estudiados son apropiados para su uso en cosméticos.

\section{E. Producción de biodiesel}

En la actualidad, existe una gran preocupación por el cambio climático y los posibles escenarios que se vivirán en unas décadas. Los combustibles fósiles desempeñan un gran rol en este tema, pues son una de las grandes causas del calentamiento global. Debido a ello, existe una necesidad urgente de crear combustibles alternativos que satisfagan la necesidad de energía; por ello, la explotación de la biomasa de diversas fuentes naturales (Kulagin et al., 2020) resulta importante. El ASM cuenta con un gran potencial para ser una alternativa viable de biocombustible, tal como lo demuestran las siguientes tres investigaciones. 
- Momoh, O. R., Audu, H. I., y Binta, Z. B. (2014). Investigating the Production of Biodiesel from Alphonso Mango Seed Oil.

Esta investigación tiene como propósito demostrar que el ASM cuenta con las propiedades necesarias para obtener de él un biocombustible que pueda ser una alternativa viable para sustituir al diésel. Tal como se observa en la tabla 11, los valores de las propiedades del biocombustible producido a partir del ASM se encuentran dentro de los parámetros establecidos por el estándar ASTM D6751. Asimismo, en la investigación se señala que, para obtener el máximo rendimiento de este combustible, los parámetros óptimos son una temperatura de $600^{\circ} \mathrm{C}$, un tiempo de reacción de una hora y una proporción de mezcla de 1:4 de ASM y metanol, respectivamente. Por lo tanto, se concluye que el ASM puede servir como insumo para producir biocombustible, ayudando a reducir la contaminación ambiental.

Tabla 11

Propiedades del biocombustible producido a partir de aceite de semilla de mango (ASM)

\begin{tabular}{lcc}
\hline \multicolumn{1}{c}{ Propiedad } & $\begin{array}{c}\text { Biocombustible } \\
\text { a partir de ASM }\end{array}$ & $\begin{array}{c}\text { ASTM D6751 } \\
\text { Estándar para biocombustibles }\end{array}$ \\
\hline Índice de cetano & 76 & 47 (mínimo) \\
Densidad $\left(\mathrm{g} / \mathrm{cm}^{3}\right)$ & 0,87 & $0,86-0,90$ \\
Punto de inflamabilidad $\left({ }^{\circ} \mathrm{C}\right)$ & 146 & 130 (mínimo) \\
Viscosidad cinemática $(\mathrm{mm} 2 / \mathrm{s})$ & 3,6 & $1,9-6,0$ \\
\hline
\end{tabular}

Fuentes: Momoh et al. (2014) y Standard Specification for Biodiesel Fuel Blend Stock (B100) for Middle Distillate Fuels (2020)

- Kumaraswamy, A., y Prasad, B.D. (2015). Performance Evaluation of Compression Ignition Direct Injection Diesel Engine on Dual Fuel Mode with Mango Oil Methyl Ester Biofuel.

El propósito de esta investigación es determinar el desempeño de los ésteres metílicos de aceite de semilla de mango (EMASM) como combustible en un motor diésel. Para ello, se llevaron a cabo diversos experimentos, en condiciones iguales, para conocer las propiedades de los siguientes combustibles: diésel, mezcla de diésel y etanol, mezcla de 20 \% EMASM y etanol, y mezcla de 100 \% EMASM y etanol. Las propiedades que se compararon fueron la eficiencia térmica, la temperatura de los gases de escape, la cantidad de emisiones de óxidos nitrosos NOx, la cantidad de emisiones de humo, la cantidad de emisiones de hidrocarburos $\mathrm{HC}$, y la cantidad de emisiones de monóxido de carbón $\mathrm{CO}$. Los 
resultados indican que los EMASM pueden ser utilizados como biocombustible en motores diésel.

- Ramalingam, S., Kaliyaperumal, A., Govindasamy, M., Ezhumalai, M., y Kumar, C. (2016). Effect of L-ascorbic Acid as Additive for Exhaust Emission Reduction in a Direct Injection Diesel Engine Using Mango Seed Methyl Ester.

Esta investigación tiene como finalidad evaluar el uso de un aditivo basado en el ácido L-ascórbico para reducir la emisión de gases de escape en un motor diésel que utiliza EMASM como biodiésel. Para ello, se realiza una comparativa de las diversas propiedades con las que cuentan el diésel puro, EMASM puro, y cuatro mezclas de distintas proporciones de ácido L-ascórbico y EMASM. Los resultados indican que las emisiones de hidrocarburos, monóxido de carbono y humo se incrementan a medida que la proporción del aditivo aumenta, pero aun así los valores resultan ser menores que los obtenidos utilizando diésel puro. Asimismo, se señala que el aditivo tiene un gran impacto al reducir las emisiones de óxidos de nitrógeno debido a que impide la formación de radicales libres. Se concluye que el ASM es una alternativa viable como biodiésel y que puede ser optimizado al utilizar un aditivo basado en ácido L-ascórbico.

\section{CONCLUSIONES}

La investigación realizada para el presente artículo no encontró revisiones de literatura sobre las aplicaciones del ASM. Sin embargo, se han encontrado diversas investigaciones que confirman el gran potencial que tiene este aceite para su utilización en diversas industrias. Por lo indicado, se consideró apropiado agrupar dichos estudios en las siguientes categorías:

A. Compuestos antimicrobianos para las industrias alimentaria y farmacéutica

B. Antioxidante natural para la industria alimentaria

C. Sustituto de la manteca de cacao

D. Cosmética

E. Producción de biodiésel

Los artículos revisados permiten concluir que el ASM:

- Presenta altos niveles de carbohidratos, calcio, potasio, magnesio y vitaminas antioxidantes, lo cual indica que podría utilizarse como fuente alternativa de estos nutrientes a través de diversos productos, como ingredientes alimentarios funcionales, antioxidantes naturales o compuestos antimicrobianos. Lo anterior implica la utilidad de un aprovechamiento de las semillas de mango en lugar de su desecho como residuo. 
- Tiene una gran capacidad antiinflamatoria y antifúngica por estar compuesto de polifenoles y ramnolípidos. Asimismo, puede resultar ser un óptimo agente antimicrobiano cuando se formula como ungüento y como cremas de uso tópico.

- Tiene un gran potencial para ser considerado un sustituto de la manteca de cacao, pues cuenta con una constitución y propiedades similares a esta última. Esto ayudaría a las industrias alimentarias a reducir sus costos, ya que la semilla de mango es una materia prima menos costosa.

- Es una alternativa viable para ser utilizado en la industria cosmética, ya que cuenta con un perfil de ácidos grasos óptimo para producir jabones y lociones, entre otros.

- Cuenta con las propiedades adecuadas para ser considerado un biocombustible, pues existe evidencia de que ha sido probado en motores diésel y ha desarrollado un comportamiento adecuado. Por ello, es un posible reemplazante de los combustibles fósiles y puede permitir una indiscutible disminución de la contaminación ambiental.

\section{REFERENCIAS}

Abdalla, A. E., Darwish, S. M., Ayad, E. H., y El-Hamahmy, R. M. (2007). Egyptian Mango By-Product 1. Compositional Quality of Mango Seed Kernel. Food Chemistry, 103(4), 1134-1140. https://doi.org/10.1016/j.foodchem.2006.10.017

Awad El-Gied, A., Abdelkareem, A., y Hamedelniel, E. (2015). Investigation of Cream and Ointment on Antimicrobial Activity of Mangifera indica Extract. Journal of Advanced Pharmaceutical Technology \& Research, 6(2), 53-57. https://doi. org/10.4103/2231-4040.154530

Ayala-Zavala, J., Vega-Vega, V., Rosas-Domínguez, C., Palafox-Carlos, H., Villa-Rodríguez, J., Siddiqui, M. W., Dávila-Aviña, J., y González-Aguilar, G. (2011). Agro-Industrial Potential of Exotic Fruit Byproducts as a Source of Food Additives. Food Research International, 44(7), 1866-1874. https://doi.org/10.1016/j.foodres.2011.02.021

Azeem, M., Nadeem, M., y Sajid, R. (2015). Analytical Characterization of Pure and Blended Watermelon (Citrulluslanatus) Oil: Impact of Blending on Oxidative Stability. Pakistan Journal of Analytical \& Environmental Chemistry, 16(1), 52-58. http://www.pjaec.pk/index.php/pjaec/article/view/127

Cirimina, R., Carnaroglio, D., Delisi, R., Arvati, S., Tamburino, A., y Pagliaro, M. (2016). Industrial Feasibility of Natural Products Extraction with Microwave Technology. Chem. Select, 3, 549-555.

Creswell, J. W., y Creswell, D. J. (2018). Research Design: Qualitative, Quantitative, and Mixed Methods Approaches (5. ${ }^{\mathrm{a}}$ ed.). SAGE Publications, Inc. 
Da Silva, A. C., y Jorge, N. (2014). Bioactive Compounds of the Lipid Fractions of Agro-Industrial Waste. Food Research International, 66, 493-500. https://doi. org/10.1016/j.foodres.2014.10.025

Diarra, S. (2014). Potential of Mango (Mangifera indica L.) Seed Kernel as a Feed Ingredient for Poultry: A Review. World's Poultry Science Journal, 70(2), 279-288. https://doi. org/10.1017/s0043933914000294

Do Nascimento Marques, N., Suzy do Nascimento Garcia, C., Yeltsin Coutinho Madruga, L., Antônio Villetti, M., de Sá Moreira de Souza Filho, M., Noriyuki Ito, E., y de Carvalho Balaban, R. (2019). Turning Industrial Waste into a Valuable Bioproduct: Starch from Mango Kernel Derivative to Oil Industry Mango Starch Derivative in Oil Industry. Journal of Renewable Materials, 7(2), 139-152. https://doi.org/10.32604/ jrm.2019.00040

Dorta, E., González, M., Lobo, M. G., Sánchez-Moreno, C., y de Ancos, B. (2014). Screening of Phenolic Compounds in By-Product Extracts from Mangoes (Mangifera indica L.) by HPLC-ESI-QTOF-MS and Multivariate Analysis for Use as a Food Ingredient. Food Research International, 57, 51-60. https://doi.org/10.1016/j. foodres.2014.01.012

Fowomola, M. A. (2010). Some Nutrients and Antinutrients Contents of Mango (Magnifera indica) Seed. African Journal of Food Science, 4(8), 472-476. https://www. researchgate.net/publication/228337486_Some_nutrients_and_antinutrients_ contents_of_mango_Magnifera_indica_seed

Jahurul, M. H. A., Zaidul, I. S. M., Nik Norulaini, N. A., Sahena, F., Kamaruzzaman, B. Y., Ghafoor, K., y Omar, A. K. M. (2014). Cocoa Butter Replacers from Blends of Mango Seed Fat Extracted by Supercritical Carbon Dioxide and Palm Stearin. Food Research International, 65, 401-406. https://doi.org/10.1016/j. foodres.2014.06.039

Jahurul, M., Zaidul, I., Ghafoor, K., Al-Juhaimi, F. Y., Nyam, K. L., Norulaini, N., Sahena, F., y Mohd Omar, A. (2015). Mango (Mangifera indica L.) By-Products and their Valuable Components: A Review. Food Chemistry, 183, 173-180. https://doi.org/10.1016/j. foodchem.2015.03.046

Jin, J., Mu, H., Wang, Y., Pembe, W., Liu, Y., Huang, J., Jin, Q., y Wang, X. (2016). Production of High-Melting Symmetrical Monounsaturated Triacylglycerol-Rich Fats from Mango Kernel Fat by Acetone Fractionation. Journal of the American Oil Chemists' Society, 94(2), 201-213. https://doi.org/10.1007/s11746-016-2929-z

Jin, J., Warda, P., Qi, C., Sun, C., Jie, L., Xie, D., Huang, J., Jin, Q., y Wang, X. (2016). Mango Kernel Fat Based Chocolate Fat with Heat Resistant Triacylglycerols: Production 
via Blending Using Mango Kernel Fat Mid-Fraction and Palm Mid-Fractions Produced in Different Fractionation Paths. RSC Advances, 6(110), 108981-108988. https://doi.org/10.1039/c6ra19438a

Karunanithi, B., Bogeshwaran, K., Tripuraneni, M., y Krishna Reddy, S. (2015). Extraction of Mango Seed Oil from Mango Kernel. International Journal of Engineering Research and Development, 11(11), 32-41. https://www.researchgate.net/ publication/342304194_Extraction_of_Mango_Seed_Oil_From_Mango_Kernel

Kathleen, P. (2010). Mango Fruit Been Found to Prevent or Stop Certain Colon and Breast Cancer Cells in the Lab. ScienceDaily, 12, 1. https://www.sciencedaily.com/ releases/2010/01/100111154926.htm

Khammuang, S., y Sarnthima, R. (2011). Antioxidant and Antibacterial Activities of Selected Varieties of Thai Mango Seed Extract. Pakistan Journal of Pharmaceutical Sciences. 24. 37-42. https://www.researchgate.net/publication/ 49714252_Antioxidant_and_antibacterial_activities_of_selected_varieties_of_ Thai_mango_seed_extract

Kim, H., Banerjee, N., Barnes, R. C., Pfent, C. M., Talcott, S. T., Dashwood, R. H., y MertensTalcott, S. U. (2016). Mango Polyphenolics Reduce Inflammation in Intestinal Colitis-Involvement of the miR-126/PI3K/AKT/mTOR Axis in vitro and in vivo. Molecular Carcinogenesis, 56(1), 197-207. https://doi.org/10.1002/mc.22484

Kittiphoom, S. (2012). Utilization of Mango Seed. International Food Research Journal, 19(4), 1325-1335. http://ifrj.upm.edu.my/19\%20(04)\%202012/5\%20IFRJ\%2019\%2004) \%202012\%20Kittiporn\%20(375).pdf

Kittiphoom, S., y Sutasinee, S. (2013). Mango Seed Oil and its Physicochemical Properties. International Food Research Journal, 20(3), 1145-1149. http://www.ifrj.upm.edu. my/20\%20(03)\%202013/15\%20IFRJ\%2020\%20(03)\%202013\%20Kittiphoom\%20 (355).pdf

Kulagin, V. A., Grushevenko, D. A., y Kapustin, N. O. (2020). Fossil Fuels Markets in the “Energy Transition" Era. Russian Journal of Economics, 6(4), 424-436. https://doi. org/10.32609/j.ruje.6.55177

Kumaraswamy, A., y Prasad, B. D. (2015). Performance Evaluation of Compression Ignition Direct Injection Diesel Engine on Dual Fuel Mode with Mango Oil Methyl Ester Biofuel. Int. J. Vehicle Structures \& Systems, 7(4), 145-148. https://doi. org/10.4273/ijvss.7.4.05

Lorenzo, J., González, R., Sánchez, M., Amado, I., y Franco, D. (2013). Effects of Natural (Grape Seed and Chestnut Extract) and Synthetic Antioxidants 
(Buthylatedhydroxytoluene, BHT) on the Physical, Chemical, Microbiological and Sensory Characteristics of Dry Cured Sausage Chorizo. Food Res. Int., 54, 611-620. http://dx.doi.org/10.1016/j.foodres.2013.07.064

Ma, X., Wu, H., Liu, L., Yao, Q., Wang, S., Zhan, R., Xing, S., y Zhou, Y. (2011). Polyphenolic Compounds and Antioxidant Properties in Mango Fruits. Scientia Horticulturae, 129(1), 102-107. https://doi.org/10.1016/j.scienta.2011.03.015

Maryam Adilah, Z., Jamilah, B., y Nur Hanani, Z. (2018). Functional and Antioxidant Properties of Protein-Based Films Incorporated with Mango Kernel Extract for Active Packaging. Food Hydrocolloids, 74, 207-218. https://doi.org/10.1016/j. foodhyd.2017.08.017

Momoh, O. R., Audu, H. I., y Binta, Z. B. (2014). Investigating the Production of Biodiesel from Alphonso Mango Seed Oil. Nigerian Journal of Technology, 33(4), 497-503. https://doi.org/10.4314/njt.v33i4.10

Mwaurah, P. W., Kumar, S., Kumar, N., Panghal, A., Attkan, A. K., Singh, V. K., y Garg, M. K. (2020). Physicochemical Characteristics, Bioactive Compounds and Industrial Applications of Mango Kernel and its Products: A Review. Comprehensive Reviews in Food Science and Food Safety, 19(5), 2421-2446. https://doi. org/10.1111/1541-4337.12598

Nadeem, M., Imran, M., Iqbal, Z., Abbas, N., y Mahmud, A. (2016). Enhancement of the Oxidative Stability of Butter Oil by Blending with Mango (Mangifera indica L.) Kernel Oil in Ambient and Accelerated Oxidation. Journal of Food Processing and Preservation, 41, 1-10. https://doi.org/10.1111/jfpp.12957

Nahar, M. K., Lisa, S. A., Nada, K., y Begum, M. (2017). Characterization of Seed Kernel Oil of Bangladeshi Mango and its Evaluation as Cosmetic Ingredient. Bangladesh Journal of Scientific and Industrial Research, 52(1), 43-48. https://doi.org/10.3329/ bjsir.v52i1.32079

Nzikou, J., Kimbonguila, A., Matos, L., Loumouamou, B., Pambou-Tobi, N., Ndangui, C., Abena, A., Silou, Th., Scher, J., y Desobry, S. (2010). Extraction and Characteristics of Seed Kernel Oil from Mango (Mangifera indica). Research Journal of Environmental and Earth Sciences, 2(1), 31-35. https://www.researchgate.net/ publication/285842805_Extraction_and_Characteristics_of_Seed_Kernel_Oil_ from_Mango_Mangifera_indica

Organización de las Naciones Unidas para la Alimentación y la Agricultura (FAO). (2020). Las principales frutas tropicales Análisis del mercado 2018. http://www.fao.org/3/ ca5692es/CA5692ES.pdf 
Rajan, S., Suganya, H., Thirunalasundari, T., y Jeeva, S. (2012). Antidiarrhoeal Efficacy of Mangifera indica Seed Kernel on Swiss Albino Mice. Asian Pacific Journal of Tropical Medicine, 5(8), 630-633. https://doi.org/10.1016/s1995-7645(12)60129-1

Ramalingam, S., Kaliyaperumal, A., Govindasamy, M., Ezhumalai, M., y Kumar, C. (2016). Effect of L-ascorbic Acid as Additive for Exhaust Emission Reduction in a Direct Injection Diesel Engine Using Mango Seed Methyl Ester. Thermal Science, 20(Suppl. 4), 999-1004. https://doi.org/10.2298/tsci16s4999r

Ribeiro, S. M. R., y Schieber, A. (2010). Bioactive Compounds in Mango (Mangifera indica L.). Bioactive Foods in Promoting Health, 34, 507-523. https://doi.org/10.1016/ b978-0-12-374628-3.00034-7

Rodríguez, E., Salangad, O., Almeda, R., Reyes, C., y Salamanez, K. (2019). Fatty Acid and Unsaponifiable Composition of Ten Philippine Food Plant Oils for Possible Nutraceutical and Cosmeceutical Applications. The Journal "Agriculture and Forestry", 65(3), 115-134. https://doi.org/10.17707/agricultforest.65.3.10

Saito, K., Kohno, M., Yoshizaki, F., y Niwano, Y. (2008). Extensive Screening for Edible Herbal Extracts with Potent Scavenging Activity Against Superoxide Anions. Plant Foods Hum. Nutr., 63, 65-70, http://dx.doi.org/10.1007/s11130-008-0071-2.

Salminen, H., Stübler, A. S., y Weiss, J. (2020). Preparation, Characterization, and Physical Stability of Cocoa Butter and Tristearin Nanoparticles Containing $\beta$-Carotene. European Food Research and Technology, 246(3), 599-608. https://doi.org/10.1007/ s00217-020-03431-0

Sathi Reddy, K., Yahya Khan, M., Archana, K., Gopal Reddy, M., y Hameeda, B. (2016). Utilization of Mango Kernel oil for the Rhamnolipid Production by Pseudomonas aeruginosa DR1 Towards its Application as Biocontrol agent. Bioresource Technology, 221, 291-299. https://doi.org/10.1016/j.biortech.2016.09.041

Sikdar, D., Hegde, S., Swamynathan, V., S, V., y Rakesh, R. (2017). Solvent Extraction of Mango (Mangifera Indica L.) Seed Kernel Oil and its Characterization. International Journal of Technical Research and Applications, 5(4), 43-47. https://www.ijtra. $\mathrm{com} /$ view/solvent-extraction-of-mango-mangifera-indica-l-seed-kernel-oiland-its-characterization.pdf

Silva, L. M. R. D., Figueiredo, E. A. T. D., Ricardo, N. M. P. S., Vieira, I. G. P., Figueiredo, R. W. D., Brasil, I. M., y Gomes, C. L. (2014). Quantification of Bioactive Compounds in Pulps and By-Products of Tropical Fruits from Brazil. Food Chemistry, 143, 398-404. https://doi.org/10.1016/j.foodchem.2013.08.001

Soccol, C. R., Costa, E. S. F. D., Letti, L. A. J., Karp, S. G., Woiciechowski, A. L., y Vandenberghe, L. P. D. S. (2017). Recent Developments and Innovations in Solid 
State Fermentation. Biotechnology Research and Innovation, 1(1), 52-71. https:// doi.org/10.1016/j.biori.2017.01.002

Sonwai, S., Kaphueakngam, P., y Flood, A. (2014). Blending of Mango Kernel Fat and Palm Oil Mid-Fraction to Obtain Cocoa Butter Equivalent. Journal of Food Science and Technology, 51(10), 2357-2369. https://doi.org/10.1007/s13197-012-0808-7

Standard Specification for Biodiesel Fuel Blend Stock (B100) for Middle Distillate Fuels (ASTM D6751-20a). (2020, August). ASTM International. https://www.astm.org/ Standards/D6751.htm

Tabio García, D., Díaz Domínguez, Y., Rondón Macias, M., Fernández Santana, E., y Piloto-Rodríguez, R. (2017). Extracción de aceites de origen vegetal. https://doi. org/10.13140/RG.2.2.11047.55201

Torres-León, C., Rojas, R., Contreras-Esquivel, J. C., Serna-Cock, L., Belmares-Cerda, R. E., y Aguilar, C. N. (2016). Mango Seed: Functional and Nutritional Properties. Trends in Food Science \& Technology, 55, 109-117. https://doi.org/10.1016/j. tifs.2016.06.009

Torres-León, C., Rojas, R., Serna-Cock, L., Belmares-Cerda, R., y Aguilar, C. N. (2017). Extraction of Antioxidants from Mango Seed Kernel: Optimization Assisted by Microwave. Food and Bioproducts Processing, 105, 188-196. https://doi. org/10.1016/j.fbp.2017.07.005

Torres-León, C., Ramírez-Guzmán, N., Ascacio-Valdés, J., Serna-Cock, L., dos Santos Correia, M. T., Contreras-Esquivel, J. C., y Aguilar, C. N. (2019). Solid-State Fermentation with Aspergillus niger to Enhance the Phenolic Contents and Antioxidative Activity of Mexican Mango Seed: A Promising Source of Natural Antioxidants. LWT - Food Science and Technology, 112, 108236. https://doi. org/10.1016/j.lwt.2019.06.003

Totani, N., Burenjargal, M., Yawata, M., y Ojiri, Y. (2008). Chemical Properties and Cytotoxicity of Thermally Oxidized Oil. Journal of Oleo Science, 57(3), 153-160. https://doi.org/10.5650/jos.57.153

Turner, R., McLean, C. H., y Silvers, K. M. (2006). Are the Health Benefits of Fish Oils Limited by Products of Oxidation? Nutrition Research Reviews, 19(1), 53-62. https://doi. org/10.1079/nrr2006117

Vieira, P. A. F., Queiroz, J. H. D., Albino, L. F. T., Moraes, G. H. K. D., Barbosa, A. D. A., Müller, E. S., y Viana, M. T. D. S. (2008). Efeitos da inclusão de farelo do resíduo de manga no desempenho de frangos de corte de 1 a 42 dias. Revista Brasileira de Zootecnia, 37(12), 2173-2178. https://doi.org/10.1590/s1516-35982008001200014 
Wu, S., Tokuda, M., Kashiwagi, A., Henmi, A., Okada, Y., Tachibana, S., y Nomura, M. (2015). Evaluation of the Fatty Acid Composition of the Seeds of Mangifera indica L. and their Application. Journal of Oleo Science, 64(5), 479-484. https://doi.org/10.5650/ jos.ess14238

Yadav, K., Garg, N., Verma, A., Kumar, S., y Trivedi, M. (2017). Optimization and Extraction of Oil from Mango Seed Kernel (Mangifera indica). Indian Journal of Agricultural Sciences, 87(7), 943-946. https://www.researchgate.net/ publication/318661900_Optimization_and_extraction_of_oil_from_mango_ seed_kernel_Mangifera_indica 
ANEXO

\begin{tabular}{|c|c|c|c|c|c|c|c|c|}
\hline N. ${ }^{\circ}$ & Año & Autores & Título & A. & B. & C. & D. & E. \\
\hline 1 & 2012 & Kittiphoom, S. & Utilization of Mango Seed. & $x$ & $x$ & $x$ & $x$ & \\
\hline 2 & 2014 & $\begin{array}{l}\text { Jahurul, M. H. A., Zaidul, } \\
\text { I. S. M., Nik Norulaini, } \\
\text { N. A., Sahena, F., } \\
\text { Kamaruzzaman, B. Y., } \\
\text { Ghafoor, K., y Omar, A. } \\
\text { K. M. }\end{array}$ & $\begin{array}{l}\text { Cocoa Butter Replacers from } \\
\text { Blends of Mango Seed Fat } \\
\text { Extracted by Supercritical } \\
\text { Carbon Dioxide and Palm } \\
\text { Stearin. }\end{array}$ & & & $x$ & & \\
\hline 3 & 2014 & $\begin{array}{l}\text { Momoh, O. R., Audu, H. } \\
\text { I., y Binta, Z. B. }\end{array}$ & $\begin{array}{l}\text { Investigating the Production } \\
\text { of Biodiesel from Alphonso } \\
\text { Mango Seed Oil. }\end{array}$ & & & & & $x$ \\
\hline 4 & 2015 & $\begin{array}{l}\text { Awad El-Gied, A., } \\
\text { Abdelkareem, A., } \\
\text { y Hamedelniel, E. }\end{array}$ & $\begin{array}{l}\text { Investigation of Cream and } \\
\text { Ointment on Antimicrobial } \\
\text { Activity of Mangifera indica } \\
\text { Extract. }\end{array}$ & $x$ & & & & \\
\hline 5 & 2015 & $\begin{array}{l}\text { Azeem, M., Nadeem, M., } \\
\text { y Sajid, R. }\end{array}$ & $\begin{array}{l}\text { Analytical Characterization } \\
\text { of Pure and Blended } \\
\text { Watermelon (Citrulluslanatus) } \\
\text { Oil: Impact of Blending on } \\
\text { Oxidative Stability. }\end{array}$ & & $x$ & & & \\
\hline 6 & 2015 & $\begin{array}{l}\text { Kumaraswamy, A., } \\
\text { y Prasad, B.D. }\end{array}$ & $\begin{array}{l}\text { Performance Evaluation of } \\
\text { Compression Ignition Direct } \\
\text { Injection Diesel Engine on } \\
\text { Dual Fuel Mode with Mango } \\
\text { Oil Methyl Ester Biofuel. }\end{array}$ & & & & & $x$ \\
\hline 7 & 2015 & $\begin{array}{l}\text { Wu, S., Tokuda, M., } \\
\text { Kashiwagi, A., Henmi, } \\
\text { A., Okada, Y., Tachibana, } \\
\text { S., y Nomura, M. }\end{array}$ & $\begin{array}{l}\text { Evaluation of the Fatty Acid } \\
\text { Composition of the Seeds of } \\
\text { Mangifera indica L. and their } \\
\text { Application. }\end{array}$ & & & & $x$ & \\
\hline 8 & 2016 & $\begin{array}{l}\text { Jin, J., Mu, H., Wang, } \\
\text { Y., Pembe, W., Liu, Y., } \\
\text { Huang, J., Jin, Q., } \\
\text { y Wang, X. }\end{array}$ & $\begin{array}{l}\text { Production of High- } \\
\text { Melting Symmetrical } \\
\text { Monounsaturated } \\
\text { Triacylglycerol-Rich Fats from } \\
\text { Mango Kernel Fat by Acetone } \\
\text { Fractionation. }\end{array}$ & $x$ & & $x$ & & \\
\hline 9 & 2016 & $\begin{array}{l}\text { Jin, J., Warda, P., Qi, C., } \\
\text { Sun, C., Jie, L., Xie, D., } \\
\text { Huang, J., Jin, Q., } \\
\text { y Wang, X. }\end{array}$ & $\begin{array}{l}\text { Mango Kernel Fat Based } \\
\text { Chocolate Fat with Heat } \\
\text { Resistant Triacylglycerols: } \\
\text { Production via Blending } \\
\text { Using Mango Kernel Fat } \\
\text { Mid-Fraction and Palm } \\
\text { Mid-Fractions Produced in } \\
\text { Different Fractionation Paths. }\end{array}$ & & & $x$ & & \\
\hline
\end{tabular}


(continuación)

\begin{tabular}{|c|c|c|c|c|c|c|}
\hline 10 & 2016 & $\begin{array}{l}\text { Ramalingam, S., } \\
\text { Kaliyaperumal, A., } \\
\text { Govindasamy, M., } \\
\text { Ezhumalai, M., y Kumar, } \\
\text { C. }\end{array}$ & $\begin{array}{l}\text { Effect of L-ascorbic Acid as } \\
\text { Additive for Exhaust Emission } \\
\text { Reduction in a Direct Injection } \\
\text { Diesel Engine Using Mango } \\
\text { Seed Methyl Ester. }\end{array}$ & & & $x$ \\
\hline 11 & 2016 & $\begin{array}{l}\text { Sathi Reddy, K., Yahya } \\
\text { Khan, M., Archana, K., } \\
\text { Gopal Reddy, M., } \\
\text { y Hameeda, B. }\end{array}$ & $\begin{array}{l}\text { Utilization of Mango Kernel } \\
\text { oil for the Rhamnolipid } \\
\text { Production by Pseudomonas } \\
\text { aeruginosa DR1 Towards } \\
\text { its Application as Biocontrol } \\
\text { agent. }\end{array}$ & $x$ & & \\
\hline 12 & 2016 & $\begin{array}{l}\text { Torres-León, C., Rojas, } \\
\text { R., Contreras-Esquivel, } \\
\text { J. C., Serna-Cock, L., } \\
\text { Belmares-Cerda, R. E., } \\
\text { y Aguilar, C. N. }\end{array}$ & $\begin{array}{l}\text { Mango Seed: Functional and } \\
\text { Nutritional Properties. }\end{array}$ & $x$ & $x$ & \\
\hline 13 & 2017 & $\begin{array}{l}\text { Kim, H., Banerjee, N., } \\
\text { Barnes, R. C., Pfent, } \\
\text { C. M., Talcott, S. T., } \\
\text { Dashwood, R. H., y } \\
\text { Mertens-Talcott, S. U. }\end{array}$ & $\begin{array}{l}\text { Mango Polyphenolics Reduce } \\
\text { Inflammation in Intestinal } \\
\text { Colitis-Involvement of the } \\
\text { miR-126/PI3K/AKT/mTOR } \\
\text { Axis in vitro and in vivo. }\end{array}$ & $x$ & & \\
\hline 14 & 2017 & $\begin{array}{l}\text { Nadeem, M., Imran, M., } \\
\text { lqbal, Z., Abbas, N., } \\
\text { y Mahmud, A. }\end{array}$ & $\begin{array}{l}\text { Enhancement of the } \\
\text { Oxidative Stability of } \\
\text { Butter Oil by Blending with } \\
\text { Mango (Mangifera indica L.) } \\
\text { Kernel Oil in Ambient and } \\
\text { Accelerated Oxidation. }\end{array}$ & & $x$ & \\
\hline 15 & 2017 & $\begin{array}{l}\text { Nahar, M. K., Lisa, S. A., } \\
\text { Nada, K., y Begum, M. }\end{array}$ & $\begin{array}{l}\text { Characterization of Seed } \\
\text { Kernel Oil of Bangladeshi } \\
\text { Mango and its Evaluation as } \\
\text { Cosmetic Ingredient. }\end{array}$ & & & $x$ \\
\hline 16 & 2017 & $\begin{array}{l}\text { Torres-León, C., Rojas, } \\
\text { R., Serna-Cock, L., } \\
\text { Belmares-Cerda, R., } \\
\text { y Aguilar, C. N. }\end{array}$ & $\begin{array}{l}\text { Extraction of Antioxidants } \\
\text { from Mango Seed Kernel: } \\
\text { Optimization Assisted by } \\
\text { Microwave. }\end{array}$ & & $x$ & \\
\hline 17 & 2018 & $\begin{array}{l}\text { Maryam Adilah, J., } \\
\text { Jamilah, B., y Nur } \\
\text { Hanani, Z. }\end{array}$ & $\begin{array}{l}\text { Functional and Antioxidant } \\
\text { Properties of Protein-Based } \\
\text { Films Incorporated with } \\
\text { Mango Kernel Extract for } \\
\text { Active Packaging. }\end{array}$ & $x$ & & \\
\hline
\end{tabular}

(continúa) 


\begin{tabular}{|c|c|c|c|c|c|c|c|c|}
\hline 18 & 2019 & $\begin{array}{l}\text { Rodriguez, E., Salangad, } \\
\text { O., Almeda, R., Reyes, C., } \\
\text { y Salamanez, K. }\end{array}$ & $\begin{array}{l}\text { Fatty Acid and Unsaponifiable } \\
\text { Composition of Ten } \\
\text { Philippine Food Plant Oils for } \\
\text { Possible Nutraceutical and } \\
\text { Cosmeceutical Applications. }\end{array}$ & $x$ & & & $x$ & \\
\hline 19 & 2019 & $\begin{array}{l}\text { Torres-León, C., } \\
\text { Ramírez-Guzmán, } \\
\text { N., Ascacio-Valdés, J., } \\
\text { Serna-Cock, L., dos } \\
\text { Santos Correia, M. T., } \\
\text { Contreras-Esquivel, J. } \\
\text { C., y Aguilar, C. N. }\end{array}$ & $\begin{array}{l}\text { Solid-State Fermentation with } \\
\text { Aspergillus niger to Enhance } \\
\text { the Phenolic Contents and } \\
\text { Antioxidative Activity of } \\
\text { Mexican Mango Seed: A } \\
\text { Promising Source of Natural } \\
\text { Antioxidants. }\end{array}$ & & $x$ & & & \\
\hline 20 & 2020 & $\begin{array}{l}\text { Mwaurah, P. W., Kumar, } \\
\text { S., Kumar, N., Panghal, } \\
\text { A., Attkan, A. K., Singh, } \\
\text { V. K., y Garg, M. K. }\end{array}$ & $\begin{array}{l}\text { Physicochemical } \\
\text { Characteristics, Bioactive } \\
\text { Compounds and Industrial } \\
\text { Applications of Mango Kernel } \\
\text { and its Products: A Review. }\end{array}$ & & $x$ & & & \\
\hline TOTAL & & & & 8 & 7 & 4 & 4 & 3 \\
\hline
\end{tabular}

Nota. A: Compuestos antimicrobianos para las industrias alimentaria y farmacéutica; B.: Antioxidante natural para la industria alimentaria; C.: Sustituto de la manteca de cacao; D.: Cosmética; E.: Producción de biodiesel. 
